Check for updates

Cite this: Soft Matter, 2017, 13, 5767

Received 29th May 2017, Accepted 20th July 2017 DOI: $10.1039 / c 7 s m 01066 d$

rsc.li/soft-matter-journal

\title{
Neutron reflectometry yields distance-dependent structures of nanometric polymer brushes interacting across water $\dagger$
}

\author{
Ignacio Rodriguez-Loureiro, ${ }^{a}$ Ernesto Scoppola, ${ }^{a}$ Luca Bertinetti, (D) ${ }^{a}$ \\ Aurelio Barbetta, ${ }^{\mathrm{ab}}$ Giovanna Fragneto ${ }^{c}$ and Emanuel Schneck (D) *a
}

\begin{abstract}
The interaction between surfaces displaying end-grafted hydrophilic polymer brushes plays important roles in biology and in many wet-technological applications. In this context, the conformation of the brushes upon their mutual approach is crucial, because it affects interaction forces and the brushes' shear-tribological properties. While this aspect has been addressed by theory, experimental data on polymer conformations under confinement are difficult to obtain. Here, we study interacting planar brushes of hydrophilic polymers with defined length and grafting density. Via ellipsometry and neutron reflectometry we obtain pressure-distance curves and determine distance-dependent polymer conformations in terms of brush compression and reciprocative interpenetration. While the pressuredistance curves are satisfactorily described by the Alexander-de-Gennes model, the pronounced brush interpenetration as seen by neutron reflectometry motivates detailed simulation-based studies capable of treating brush interpenetration on a quantitative level.
\end{abstract}

\section{Introduction}

Nanometric brushes of end-grafted polymers are widely used in wet- and biotechnology. Applications include the biocompatible functionalization of nanoparticles and material surfaces, ${ }^{1}$ the stabilization of colloidal suspensions ${ }^{2}$ and foams, ${ }^{3}$ as well as shear lubrication. ${ }^{4}$ In most cases the forces between two interacting brushes are responsible for their function, where the forces both perpendicular ${ }^{2,3}$ or parallel $^{4}$ to the brush-grafting surfaces can play the principal role. Both are relevant also for the saccharide-based polymer brushes found on the surfaces of biological membranes, ${ }^{5,6}$ as they affect, for instance, the interaction between adjacent Gram-negative bacteria in biofilms, and in turn biofilm mechanical properties. A pivotal aspect of the interaction of two brushes is the polymer conformation in terms of compression and mutual interpenetration. The latter has immediate consequences for the brushes' shear tribological properties, because shear stress between sliding polymer brushes is known to depend on the level of brush interpenetration. ${ }^{7}$ The interaction of polymer brushes has been

\footnotetext{
${ }^{a}$ Max Planck Institute of Colloids and Interfaces, Am Mühlenberg 1, 14476 Potsdam, Germany. E-mail: schneck@mpikg.mpg.de; Fax: +49-331567-9402; Tel: +49-331567-9404

${ }^{b}$ Institut de Chimie Séparative de Marcoule, 30207 Bagnols sur Cèze, France

${ }^{c}$ Institut Laue-Langevin, 71 avenue des Martyrs, 38000 Grenoble, France

$\dagger$ Electronic supplementary information (ESI) available. See DOI: 10.1039/c7sm01066d
}

extensively studied theoretically ${ }^{8-11}$ and computationally, ${ }^{7,12-14}$ both in terms of pressure-distance curves (i.e., distance-dependent interaction pressures) and in terms of brush conformations. Pressure-distance curves have been determined also experimentally, using amphiphilic multilayers, ${ }^{15}$ surface force apparatus $(\mathrm{SFA})^{16}$ and atomic force microscopy (AFM). ${ }^{17}$ In contrast, experimental data on the conformation of interacting brushes has remained sparse ${ }^{18,19}$ despite its great technological and biological importance.

Insight into structures "buried" between two surfaces or interfaces, such as the conformation of polymer brushes under confinement, is generally difficult to obtain experimentally. At first, neither of the two surfaces is accessible by scanning near-field techniques like AFM, because this is prevented by the presence of the respective counterpart. Secondly, the relevant structures are of the order of 0.1-100 nm; these small length scales cannot be resolved by optical microscopy and at the same time impede the use of fluorescent labels, therefore excluding complementary techniques like fluorescence resonance energy transfer (FRET). Finally, the structural features of interest are easily perturbed and sensitive to thermodynamic conditions, rendering all cryo-based techniques inadequate. $\mathrm{X}$-ray and neutron scattering are among the very few techniques that can probe such structures with the required subnanometer spatial resolution. They can be used in a wide pressure and temperature range and provide sample-averaged structural information. Neutron scattering, apart from being 
truly non-destructive, has the unique advantage of contrast variation: chemical components of interest can be highlighted by selective deuteration (i.e., replacing hydrogen with deuterium) without changing their chemistry. ${ }^{20}$ Specular neutron reflectometry (NR) with contrast variation unambiguously reveals matter density profiles perpendicular to an interface, ${ }^{21,22}$ but requires macroscopically-large planar samples with near-perfect flatness and very low surface roughness. While the fabrication of single surfaces meeting these requirements is well established to date, bringing two such surfaces into a defined interaction distance over large areas has remained a challenging task. In their pioneering studies, Kuhl and co-workers carried out NR measurements while keeping two brush-decorated solid substrates parallel at separations down to below $100 \mathrm{~nm} .^{18}$ Using this approach, they determined the density profiles of comparatively thick polystyrene (PS) brushes interacting across toluene for two grafting surface separations. Later, in order to unambiguously determine the degree of brush interpenetration, one of the PS brushes was deuterated (dPS) while the other one was hydrogenated (hPS). ${ }^{19}$

Here, we study the interaction of hydrophilic polymer brushes across aqueous media. For this purpose, we make use of an architecture in which two planar amphiphilic monolayers act as grafting surfaces for the polymer brushes and are brought into contact at distances as small as few tens of $\mathrm{nm}$ and less. These are the typical length scales relevant for the interaction of polymer brushes on biological surfaces ${ }^{5,6}$ and of polymer-decorated nanoparticles in biotechnology. ${ }^{23} \mathrm{Via}$ controlled dehydration our double-monolayer configuration enables the tunable approach of the two surfaces and the separation-dependent structural investigation. ${ }^{24}$ The monolayers are composed of lipids and lipopolymers (Fig. 1A), supported by a hydrophobized planar solid on one side, but free-standing on the other side (Fig. 1B and C). With that, a homogeneous surface separation on the planar substrate is readily realized, in contrast to approaches involving two planar solids, where creation of a defined interaction distance is generally difficult. ${ }^{25}$ We use ellipsometry to obtain the pressuredistance curves of interacting brushes of various polymerization degrees and grafting densities. For selected brushes, we use NR to determine the brush conformation as a function of the grafting surface separation. To this end we describe the reflectivity curves in a wide separation range with a common model based on the volume fraction distributions of all chemical components and their distance dependence. We find that the pressure-distance curves are surprisingly well described by the Alexander-de-Gennes (AdG) model. ${ }^{8,9}$ In contrast, NR reveals pronounced brush interpenetration captured only by the most
A

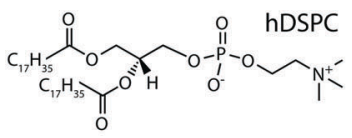

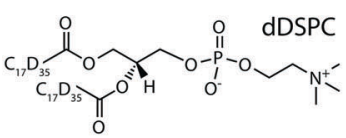

B
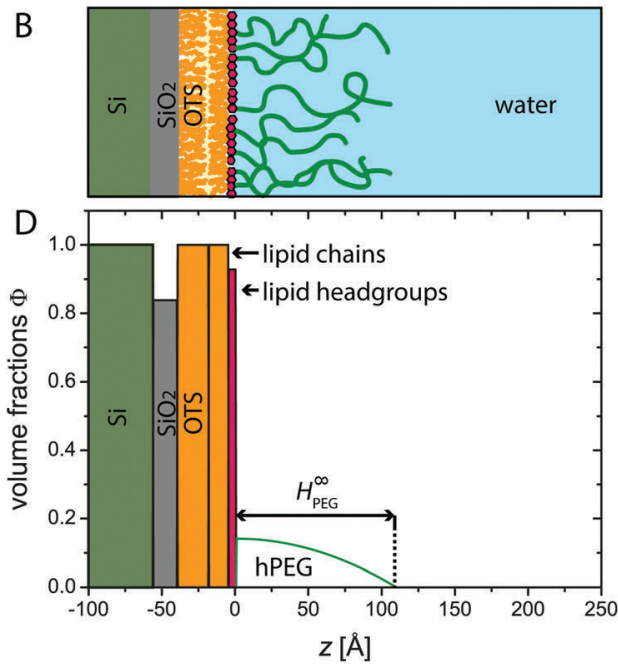
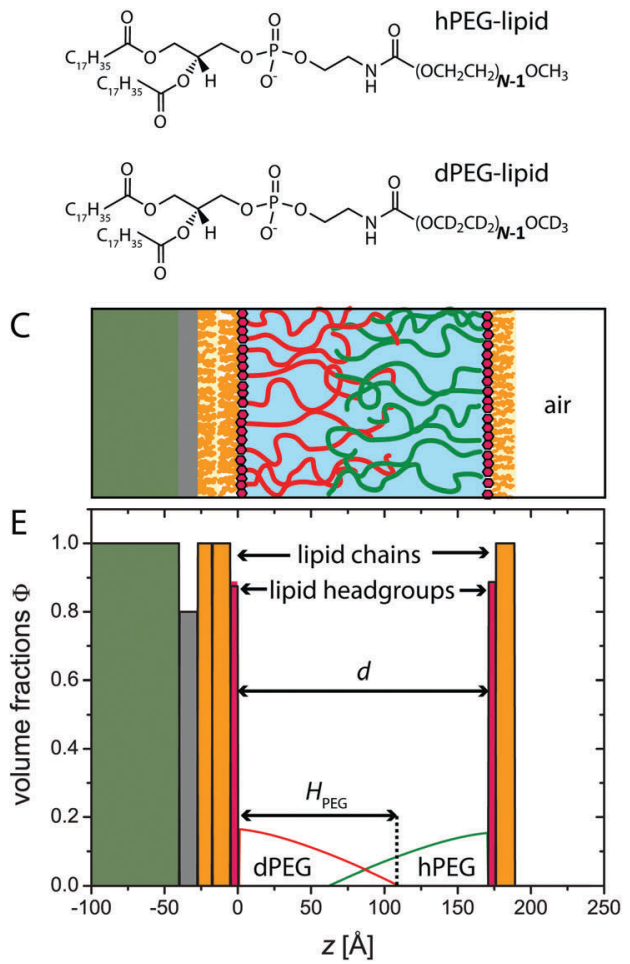

Fig. 1 (A) Chemical structures of the amphiphilic molecules DSPC (1,2-distearoyl-sn-glycero-3-phosphocholine) and PEG-lipid (1,2-distearoyl-snglycero-3-phosphoethanolamine- $N$-[methoxy(polyethylene glycol)]). Hydrogenous and chain-deuterated versions of DSPC are denoted as hDSPC and dDSPC, respectively. PEG-lipid versions with hydrogenous and deuterated PEG chains are denoted as hPEG-lipid and dPEG-lipid. (B) Schematic illustration of a single uncompressed lipid-anchored brush in water. (C) Schematic illustration of a double-monolayer architecture with two interacting lipid-anchored PEG brushes. Distal lipid chains and the proximal PEG brush are deuterated while the proximal lipid chains and the distal PEG brush are hydrogenous. The solid surface is hydrophobically functionalized with octadecyltrichlorosilane (OTS). (D and E) Simplified representations of the spatial distributions of all components ( $\mathrm{Si}, \mathrm{SiO}_{2}$, OTS, lipid chains, lipid headgroups, and $\mathrm{PEG}$ ) in panels $\mathrm{B}$ and $\mathrm{C}$, respectively. 
rigorous simulation-based theoretical descriptions of interacting polymer brushes.

\section{Materials and methods}

\subsection{Materials and sample preparation}

Unless stated otherwise, all chemicals were purchased from Sigma (St. Louis, MO, USA) and used without further purification. $\mathrm{D}_{2} \mathrm{O}(99 \%)$ was purchased from Euriso-Top, Saint-Aubin, France. PEG brushes - either in hydrogenous form (hPEG) or fully deuterated (dPEG) - were prepared from mixtures of two lipids, whose chemical structures are shown in Fig. 1A: (i) distearoyl-snglycero-3-phosphoethanolamine lipids with a PEG chain comprising $22 \leq N \leq 114$ monomers attached to the hydrophilic headgroup (hPEG-lipid or dPEG-lipid); (ii) 1,2-distearoyl-sn-glycero-3phosphocholine (DSPC), either in hydrogenous form (hDSPC) or with fully deuterated alkyl chains (dDSPC). DSPC and PEGlipid were purchased from Avanti Polar Lipids (Alabaster, AL, USA). Silicon single (111) crystal blocks of $50 \mathrm{~mm} \times 50 \mathrm{~mm} \times$ $10 \mathrm{~mm}$ or $50 \mathrm{~mm} \times 40 \mathrm{~mm} \times 10 \mathrm{~mm}$ size, polished on one large face, were purchased from Synchrotronix (Annemasse, France). The polished surface was covered with a thin layer of native amorphous silicon oxide (SiO2). Silicon wafers $(150 \mathrm{~mm}$ diameter, $625 \mu \mathrm{m}$ thickness) of which the polished surface was covered with a $105 \mathrm{~nm}$ layer of thermal silicon oxide were purchased from SIEGERT Wafer GmbH (Aachen, Germany) and cut into rectangular pieces of $20 \mathrm{~mm} \times 10 \mathrm{~mm}$. Silicon blocks and pieces were cleaned by washing with organic solvents (chloroform, acetone, and ethanol) and UV-ozone treatment. They were then rendered hydrophobic via covalent functionalization with octadecyltrichlorosilane (OTS) by immersion in freshly prepared solutions of OTS in hexadecane at a concentration of $1 \mathrm{mM}$ for $1 \mathrm{~h}$ and subsequent rinsing in hexadecane and ethanol. Lipid monolayers anchoring the PEG brushes were deposited onto the functionalized substrates utilizing a combination of the Langmuir-Schaefer (LS) and Langmuir-Blodgett (LB) techniques. ${ }^{26}$ For this purpose, mixtures of DSPC (either hDSPC or dDSPC) and PEG-lipid (either hPEG-lipid or dPEG-lipid), with PEG-lipid mole fractions $f$ ranging from $0 \%$ to $10 \%$, in chloroform at $2 \mathrm{mg} \mathrm{ml}^{-1}$ overall concentration were prepared and spread at the air-water interface in a Teflon Langmuir trough (Nima Technology, Coventry, UK) containing $\mathrm{H}_{2} \mathrm{O}$. After compression to a lateral pressure of $\Pi_{\text {lat }}=35 \mathrm{mN} \mathrm{m}^{-1}$, a first monolayer was transferred onto the hydrophobic OTS by LS, i.e., with the solid surface facing the water surface. This procedure results in single uncompressed brushes exposed to bulk water (see Fig. 1B). No significant change in $\Pi_{\text {lat }}$ upon substrate/ monolayer contact was observed, evidencing a transfer ratio close to $100 \%$, which is typical for the LS technique. Selected samples were characterized by NR already at this stage, in order determine their structure (see Results section). In all other cases, the Si block was then rotated under water by $90^{\circ}$, so that its surface ended up perpendicular to the water surface. The remaining lipid monolayer at the air/water interface was then removed and replaced with a fresh monolayer compressed to $\Pi_{\text {lat }}=35 \mathrm{mN} \mathrm{m}^{-1}$. This second monolayer was then transferred by LB, i.e., by pulling the block upwards. As a result, the PEG brushes anchored to the monolayers face each other as schematically illustrated in Fig. 1C. With this combination of LS and LB highly structured sample architectures were achieved (see Results section). Alternative sample preparations by the commonly used double LB deposition were less successful (see ESI $\dagger$ ).

\subsection{Determination of pressure-distance curves by ellipsometry}

Ellipsometry enables the characterization of interfacial layers in terms of refractive indices and thicknesses. The method is based on the change in the polarization state of light upon reflection from the surface. For a given refractive index $n$, the change depends on the layer thickness and is quantified in terms of the phase difference $\Delta$ and the amplitude ratio $\Psi$ encoded in the ratio between the complex reflection coefficients $R_{\mathrm{s}}$ and $R_{\mathrm{p}}$ for $\mathrm{s}$ and p polarizations, respectively: ${ }^{27}$

$$
R_{\mathrm{p}} / R_{\mathrm{S}}=\tan \Psi \mathrm{e}^{-i \Delta}
$$

For ellipsometry measurements, silicon chips with thermal oxide were used as substrates for the double monolayers.
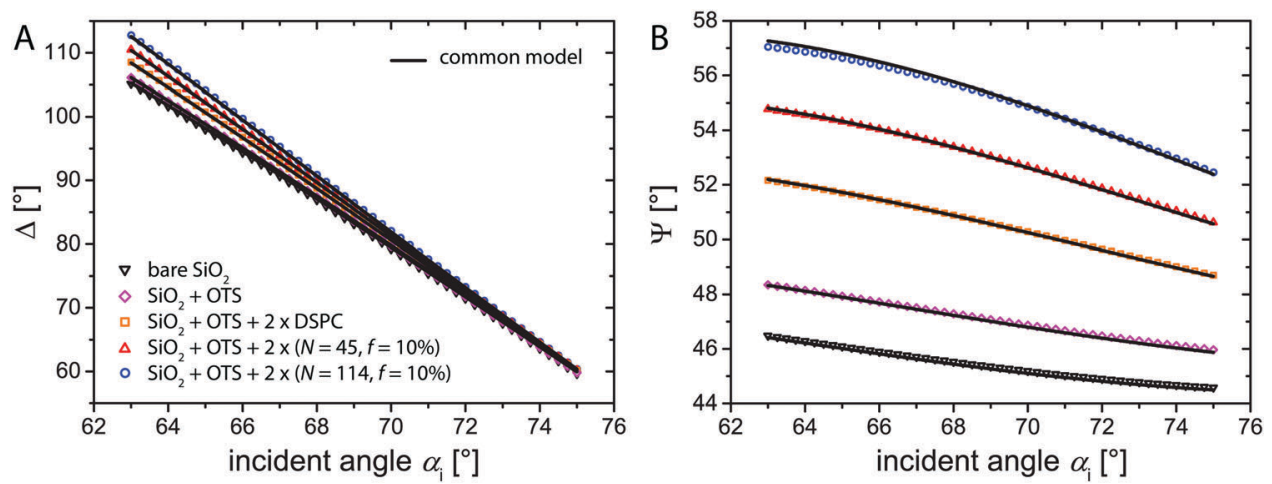

Fig. 2 Ellipsometric angles $\Delta$ (A) and $\Psi$ (B) as functions of the incident angle $\alpha_{\mathrm{i}}$ for various samples under fully dehydrated conditions: (i) bare SiO (ii) $\mathrm{SiO}_{2}$ with OTS, (iii) $\mathrm{SiO}_{2}$ with OTS and interacting DSPC monolayers, and (iv) $\mathrm{SiO}_{2}$ with OTS and interacting DSPC monolayers displaying brushes with $f=10 \%$ and $N=45$ or $N=114$. Statistical errors are smaller than the symbol size. Solid lines indicate the bets-matching combined fits to $\Delta$ and $\Psi$ for each sample. 
Silicon has the complex refractive index $n_{\mathrm{Si}}=3.885-0.018 \mathrm{i}^{28}$ Measurements were performed by scanning the incident angle $\alpha_{i}$ from $63^{\circ}$ to $75^{\circ}$, with an Optrel Multiskop ellipsometer working with a wavelength $\lambda_{\text {elli }}=632.8 \mathrm{~nm}$. The best fit to the results on bare substrates yielded a $\mathrm{SiO}_{2}$ refractive index of $n_{\mathrm{SiO}_{2}}=1.468$, close to the literature value ${ }^{29}$ and an oxide layer thickness of $105.5 \mathrm{~nm}$. In the next step, the measurement values obtained for fully dehydrated samples $\left(h_{\text {rel }}<5 \%\right.$, achieved by streaming with $\mathrm{N}_{2}$ ) were modeled while accounting for the above-defined oxide layer properties, to obtain the thickness $D_{\text {org }}$ of the dry organic layer jointly formed by OTS and the two brush-decorated lipid monolayers. In this procedure, the best fit was obtained with a refractive index $n_{\mathrm{org}}=1.5$, consistent with earlier reports on organic materials. ${ }^{30-32}$ Fig. 2 shows the two ellipsometric angles, $\Delta$ and $\Psi$, as functions of $\alpha_{i}$ for various dry samples. The solid lines indicate simultaneous fits to both ellipsometric angles and correspond to organic layer thicknesses of $D_{\text {org }}=2.1 \mathrm{~nm}, D_{\text {org }}=7.4 \mathrm{~nm}, D_{\text {org }}=8.6 \mathrm{~nm}$, and $D_{\text {org }}=10.7 \mathrm{~nm}$ for bare OTS, for OTS with interacting DSPC monolayers ( $f=0 \%$ ), and for OTS with interacting DSPC monolayers displaying brushes with $f=10 \%$ and $N=45$ or $N=114$, respectively. This systematic increase is remarkably proportional to the material amount that is added according to the doublemonolayer architecture (see ESI $\dagger$ ).

In the last step, the ellipsometric angles obtained at controlled humidity $h_{\text {rel }}$ were modeled while accounting for the known optical parameters of oxide and dry organic layers. The humidity-dependent equivalent thickness (see eqn (5) below) of the water layer, $D_{\mathrm{w}}\left(h_{\text {rel }}\right)$, was then determined assuming $n_{\mathrm{w}}=1.33$ for the refractive index of water. As shown in the ESI, $\dagger$ the position of the water layer with respect to the organic layer in the model has negligible influence on the results. This is because in the thin-film limit ellipsometry is merely sensitive to the overall optical path. In order to instantly follow the evolution of $h_{\text {rel }}$, these measurements were performed at a fixed incident angle of $\alpha_{i}=70^{\circ}$. Note that measurements at a single incident angle are sufficient to determine the incremental change of a layer thickness when this is the only unknown parameter. Humidity was controlled by placing the samples inside a closed chamber through which humidified $\mathrm{N}_{2}$ was streamed. The gas was humidified by letting it pass through a temperature-controlled water bath in the form of mm-sized bubbles. High humidities were realized by elevating the water temperature to close to or even slightly above the sample temperature. Low humidities were realized either by lowering the bath temperature or by mixing the humidified $\mathrm{N}_{2}$ stream with dry $\mathrm{N}_{2}$. The humidity at the sample position was measured with a calibrated humidity sensor (Sensirion SHT75, measurement uncertainty $\Delta h_{\text {rel }}= \pm 2 \%$ ), placed close to the sample surface.

\subsection{Structural investigation by neutron reflectometry}

2.3.1 Setup and experiments. Neutron reflectometry (NR) measurements were performed on the D17 time-of-flight (TOF) reflectometer at the Institut Laue-Langevin (ILL, Grenoble, France). The intensity of the reflected neutron beam relative to the intensity of the incident beam was recorded as a function of the component of the scattering vector normal to the interface, $q_{z}=(4 \pi / \lambda) \sin \theta_{\mathrm{i}}$, where $\lambda$ is the neutron wavelength and $\theta_{\mathrm{i}}$ the incident angle. Measurements were carried out at two fixed angles of incidence, $\theta_{\mathrm{i}}=0.8 \mathrm{deg}$ and $\theta_{\mathrm{i}}=3.2 \mathrm{deg}$ using a wavelength range of $2 \AA<\lambda<22 \AA$. The relative resolution in $q_{z}, \Delta q_{z} / q_{z}$, defined via the full width at half maximum (FWHM) was $q_{z}$-dependent and varied in the range $2 \%<\Delta q_{z} / q_{z}<14 \%$. While modeling experimental reflectivity curves the finite experimental resolution was taken into account by convoluting the initial reflectivity curves, calculated for the case of infinite resolution, with Gaussian functions representing the resolution function of the experiments. The reflectivity curves displaying the reflected intensity as a function of $q_{z}$ (see Fig. 3A and 5A) depend on the depth profile of the neutron scattering length density (SLD) $\rho$ across the interface between the two bulk media, Si on one side and water or humidified air, respectively, on the other side. The SLD of a homogeneous medium depends on the density $n_{i} / v$ of the constituting nuclides and on their coherent scattering lengths $b_{i}$ :

$$
\rho=\sum_{i} \frac{n_{i}}{v} b_{i}
$$

where $n_{i}$ is the number of nuclides $i$ in a volume $v$.
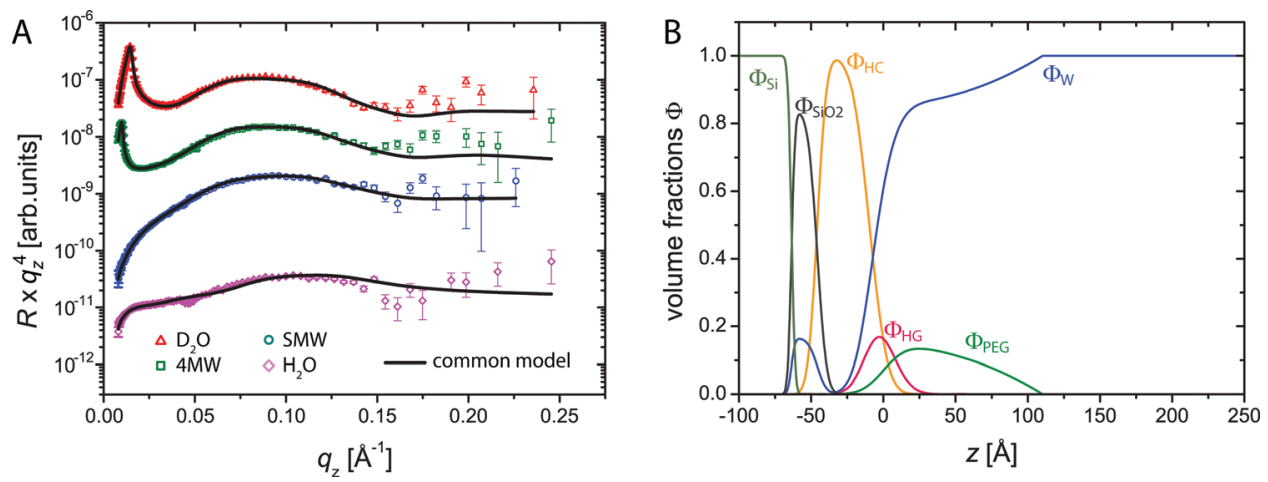

Fig. 3 (A) Neutron reflectivity curves (symbols) of a single, uncompressed brush with $f=10 \%$ and $N=114$ obtained with the four water contrasts $\mathrm{D}_{2} \mathrm{O}$, $4 \mathrm{MW}, \mathrm{SMW}$, and $\mathrm{H}_{2} \mathrm{O}$ (see Methods section). Solid lines indicate the theoretical reflectivity curves according to the best-matching parameters in the common model. The reduced chi-square deviation is $\chi_{\text {red }}{ }^{2}=5.5$. (B) Corresponding volume fraction profiles $\Phi$ of $\mathrm{Si}_{\text {, }} \mathrm{SiO}_{2}$, hydrocarbon chains of OTS and lipids (HC), lipid headgroups (HG), PEG, and water (W). 
Single uncompressed brushes exposed to bulk water (see Fig. 1B) were characterized in several contrast fluids obtained by mixing $\mathrm{H}_{2} \mathrm{O}$ and $\mathrm{D}_{2} \mathrm{O}$ at defined mixing ratios leading to distinct SLDs. $\mathrm{H}_{2} \mathrm{O}$ and $\mathrm{D}_{2} \mathrm{O}$ have SLDs of $\rho_{\mathrm{w}}=-0.56 \times 10^{-6} \AA^{-2}$ and $\rho_{\mathrm{w}}=6.36 \times 10^{-6} \AA^{-2}$, respectively. In addition, we used $\mathrm{H}_{2} \mathrm{O} / \mathrm{D}_{2} \mathrm{O}$ mixtures having $\rho_{\mathrm{w}}=4.0 \times 10^{-6} \AA^{-2}(4 \mathrm{MW})$ and with $\rho_{\mathrm{w}}=2.07 \times 10^{-6} \AA^{-2}$, the latter known as silicon-matched water or SMW since its SLD matches that of the silicon substrate. For interacting brushes (see Fig. 1C) the relative humidity was $\mathrm{H}_{2} \mathrm{O}$-based and was varied using the newest generation humidity cells of D17 using Peltier elements to warm up the sample and a water reservoir thermally isolated from the sample, and a temperature-controlled water bath to cool down. High humidities were realized by elevating the water temperature to close to or even slightly above the sample temperature. Reflectivity curves were measured in loops of $5 \mathrm{~min}$ acquisitions, to capture also conditions of extremely high humidity which are difficult to maintain over longer periods. ${ }^{33,34}$

2.3.2 Reflectivity data analysis. The reflectivity data were analyzed in the spirit of our recent work on polymer brushes in contact with protein solutions: $:^{35,36}$ the samples are described by common models accounting for all measurement conditions. For a single uncompressed brush in water (see Fig. 1B) the corresponding model comprises measurements in all contrast fluids. For interacting brushes subject to controlled dehydration (see Fig. 1C), the corresponding model accounts for all hydration levels characterized by their respective grafting surface separations $d$. Each model describes the volume fractions $\Phi_{\mathrm{i}}$ of all compounds, i.e., silicon ( $\mathrm{i}=$ "Si"), silicon oxide $\left(\mathrm{i}=\right.$ "SiO ${ }_{2}$ "), OTS $(\mathrm{i}=$ "OTS"), lipid chains $(\mathrm{i}=$ "CH”), lipid headgroups ( $\mathrm{i}=$ "HG”), PEG ( $\mathrm{i}=$ "PEG”), and water $(\mathrm{i}=$ " "W") as functions of the depth coordinate $z$. It incorporates several adjustable parameters that are fitted simultaneously to the results obtained under different conditions. Simplified schematic illustrations of single and interacting brushes are presented in Fig. 1D and E.

The SLD profile for a single uncompressed brush is given by:

$$
\begin{aligned}
\rho(z)= & \Phi_{\mathrm{Si}}(z) \rho_{\mathrm{Si}}+\Phi_{\mathrm{SiO}_{2}}(z) \rho_{\mathrm{SiO}_{2}}+\Phi_{\mathrm{OTS}}(z) \rho_{\mathrm{OTS}}+\Phi_{\mathrm{CH}}(z) \rho_{\mathrm{CH}} \\
& +\Phi_{\mathrm{HG}}(z) \rho_{\mathrm{HG}}+\Phi_{\mathrm{PEG}}(z) \rho_{\mathrm{PEG}}+\Phi_{\mathrm{w}}(z) \rho_{\mathrm{w}}
\end{aligned}
$$

where $z$ denotes the distance measured along the normal to the planar sample surface. The brush grafting surface on the solid substrate is at $z=0$ and coincides with the interface between lipid headgroups and the aqueous region (Fig. 1D). When a second brush is deposited on top of the first one then the second grafting surface is located at $z=d$ (Fig. 1E). Selective deuteration of lipid chains and PEG portions (Fig. 1A) further allows distinguishing between proximal and distal chain and PEG distributions ( $\mathrm{i}=$ "CH,P”, $\mathrm{i}=$ = "CH,D”, $\mathrm{i}=$ "PEG,P”, and $\mathrm{i}=$ "PEG,D”, respectively), leading to the following description of the SLD profile for two interacting brushes:

$$
\begin{aligned}
\rho(z)= & \Phi_{\mathrm{Si}}(z) \rho_{\mathrm{Si}}+\Phi_{\mathrm{SiO}_{2}}(z) \rho_{\mathrm{SiO}_{2}}+\Phi_{\mathrm{OTS}}(z) \rho_{\mathrm{OTS}}+\left(\Phi_{\mathrm{HG}, \mathrm{P}}(z)\right. \\
& \left.+\Phi_{\mathrm{HG}, \mathrm{D}}(z)\right) \rho_{\mathrm{HG}}+\Phi_{\mathrm{w}}(z) \rho_{\mathrm{w}}+\Phi_{\mathrm{CH}, \mathrm{P}}(z) \rho_{\mathrm{CH}, \mathrm{P}}+\Phi_{\mathrm{CH}, \mathrm{D}}(z) \rho_{\mathrm{CH}, \mathrm{D}} \\
& +\Phi_{\mathrm{PEG}, \mathrm{P}}(z) \rho_{\mathrm{PEG}, \mathrm{P}}+\Phi_{\mathrm{PEG}, \mathrm{D}}(z) \rho_{\mathrm{PEG}, \mathrm{D}}
\end{aligned}
$$

where we also distinguish between proximal and distal headgroups ( $\mathrm{i}$ = "HG,P” and i = "HG,D”, respectively), although they have the same SLD. To quantify the amount per area of each component (except the bulk media) it is convenient to introduce the equivalent thickness

$$
D_{\mathrm{i}}=\int_{-\infty}^{\infty} \Phi_{\mathrm{i}}(z) \mathrm{d} z,
$$

which can be viewed as the thickness of an equivalent layer entirely composed of component $\mathrm{i}$. The profiles of $\mathrm{Si}, \mathrm{SiO}_{2}$, OTS, and lipid regions (HC chains and headgroups), are treated with a slab model, in which the crystalline silicon substrate is represented by a semi-infinite continuum with fixed scattering length density $\rho_{\mathrm{Si}}=2.07 \times 10^{-6} \AA^{-2}$. The $\mathrm{SiO}_{2}$, OTS, lipid chain and lipid headgroup layers are represented as homogeneous slabs with adjustable thicknesses $d_{\mathrm{SiO}_{2}}, d_{\mathrm{OTS}}, d_{\mathrm{CH}}$, and $d_{\mathrm{HG}}$, respectively (Fig. 1D and E). To account for interfacial roughness, the profiles of all slabs are modulated by error functions with adjustable roughness parameters $\zeta_{\mathrm{i}}$. The SLD of silicon oxide is fixed at the literature value, $\rho_{\mathrm{SiO}_{2}}=3.4 \times 10^{-6} \AA^{-2}$. The water fraction (in the form of hydration layers or silanols) in the $\mathrm{SiO}_{2}$ layer, $\Phi_{\mathrm{w}}^{\mathrm{SiO}_{2}}$, is an adjustable parameter. The SLD of OTS is allowed to vary between $-0.5 \times 10^{-6} \AA^{-2}$ and 0 , and the water content in the OTS and lipid chain layers is set to 0 , due to their hydrophobic character. To model the lipid layers we use the SLD and volume values reported in ref. 37. Chains of the proximal lipids are all hydrogenous, so that $\rho_{\mathrm{CH}}$ (eqn (3)) and $\rho_{\mathrm{CH}, \mathrm{P}}($ eqn (4)) are set to the value for hydrogenous chains, $\rho_{\text {hCH }}=-0.4 \times 10^{-6} \AA^{-2}$ (ref. 37). In the distal monolayer the fraction of hydrogenous chains (those of dPEG-lipid) is $f$, while the fraction of deuterated chains (those of dDSPC, with $\rho_{\mathrm{dCH}}=$ $7.07 \times 10^{-6} \AA^{-2}$, ref. 37) is $(1-f)$. For the distal monolayer, we therefore have $\rho_{\mathrm{CH}, \mathrm{D}}=f \rho_{\mathrm{hCH}}+(1-f) \rho_{\mathrm{dCH}}$ and for $f=10 \%$ we numerically obtain $\rho_{\mathrm{CH}, \mathrm{D}}=6.326 \times 10^{-6} \AA^{-2}$. The headgroup SLD was set to $\rho_{\mathrm{HG}}=1.75 \times 10^{-6} \AA^{-2} \cdot{ }^{37}$ These SLD values are calculated from chain and headgroup volumes of DSPC (and of the DSPE portion of PEG-lipid to good approximation), $v_{\mathrm{CH}}=$ $980 \AA^{3}$ and $v_{\mathrm{HG}}=344 \AA^{3}$, respectively. ${ }^{37}$ The headgroup amount per area in terms of the equivalent thickness is therefore coupled to the corresponding chain equivalent thickness via $D_{\mathrm{HG}}=D_{\mathrm{CH}} v_{\mathrm{HG}} / v_{\mathrm{CH}}=0.35 D_{\mathrm{CH}}$. For a given $D_{\mathrm{HG}}$, the water fraction in the headgroup slab then follows as $\Phi_{\mathrm{w}}^{\mathrm{HG}}=1-d_{\mathrm{HG}} / D_{\mathrm{HG}}$ (compare eqn (7) below). In the distal lipid monolayer, all roughnesses (between water/headgroup, headgroup/chain and chain/air) are assumed to be identical and described by a single parameter $\zeta_{\mathrm{D}}$. This approximation is justified when the roughness is dominated by the interfacial fluctuations, which are conformal. The SLDs of the proximal and distal PEG brushes are set to the literature values of hydrogenous PEG $\left(\rho_{\text {hPEG }}=0.6 \times\right.$ $10^{-6} \AA^{-2}$, ref. 38) or deuterated PEG $\left(\rho_{\text {dPEG }}=7.0 \times 10^{-6} \AA^{-2}\right)$, respectively: $\rho_{\mathrm{PEG}}=\rho_{\mathrm{hPEG}}\left(\right.$ in eqn (3)), $\rho_{\mathrm{PEG}, \mathrm{D}}=\rho_{\mathrm{hPEG}}($ in eqn (4)), and $\rho_{\text {PEG,P }}=\rho_{\text {dPEG }}$ (eqn (4)). These SLD values imply an ethylene glycol monomer volume of $v_{\mathrm{EG}}=69 \AA^{3}$. The PEG amount per area in terms of the equivalent thickness is therefore also coupled to the corresponding chain equivalent thickness via $D_{\mathrm{PEG}}=D_{\mathrm{CH}} f N v_{\mathrm{EG}} / v_{\mathrm{CH}}$. For the single uncompressed brush, the 
nominal values of $\rho_{\mathrm{W}}$ for $\mathrm{D}_{2} \mathrm{O}, 4 \mathrm{MW}, \mathrm{SMW}$, and $\mathrm{H}_{2} \mathrm{O}$ are used, however allowing for small variations around these nominal values to account for imperfect exchange during the rinsing process. For interacting brushes exposed to $\mathrm{H}_{2} \mathrm{O}$-based humidity $\rho_{\mathrm{W}}$ is fixed to the nominal value of $\mathrm{H}_{2} \mathrm{O}$.

The profile of the PEG brushes is based on a truncated power law description:

$$
\Phi_{\mathrm{PEG}}(z)=I(z) \Phi_{0}\left(1-\left(\frac{z}{H_{\mathrm{PEG}}}\right)^{n}\right) . \quad z \leq H_{\mathrm{PEG}}
$$

$I(\mathrm{z})$ represents the smearing of the profile at the rough grafting surface which has the shape of an error function with roughness $\zeta$ (see above). $H_{\mathrm{PEG}}$ and $\Phi_{0}$ denote the brush extension and the maximal PEG volume fraction at the grafting surface, respectively. For the single uncompressed PEG brush, the exponent is set as $n=2$ to generate the parabolic profiles (Fig. 1D) predicted by analytical SCF theory ${ }^{39}$ and confirmed experimentally. ${ }^{36}$ For the interacting brushes under compression, $\Phi_{\mathrm{PEG}, \mathrm{P}}$ is described by eqn (6) while $\Phi_{\mathrm{PEG}, \mathrm{D}}$ is described by a mirrored version of eqn (6) shifted along the $z$-axis by the grafting surface separation $d$ (Fig. 1E). Moreover, $n$ is allowed to vary when the brushes get compressed, as previously suggested. ${ }^{19}$ Both profiles are truncated at the opposing brush grafting surface in cases when $H_{\mathrm{PEG}} \gtrsim d$. Finally, the water profile followed from the requirement

$$
\sum_{j} \Phi_{i}(z) \equiv 1
$$

which in our model for the interacting brushes only holds for all $z$-values up to the water-free chain layer of the distal lipid monolayer, because we do not account for the air volume fraction explicitly (see eqn (4)). Most of the model parameters are plausibly assumed to be independent of the surface separation $d$ : The SLDs of all components, the proximal and distal lipid and PEG amounts, as well as the roughness, thicknesses, and water fractions of $\mathrm{SiO}_{2}$, OTS, and proximal lipid chain and headgroup slabs. However, the most interesting quantities, namely those concerning the brush conformation and the configuration of the distal lipid monolayer, are allowed to vary with $d$. The $d$-dependence of the brush extension $H_{\mathrm{PEG}}$ is modeled with an exponential saturation function

$$
H_{\mathrm{PEG}}(d)=H_{\mathrm{PEG}}^{\infty} \cdot\left[1-\exp \left(-d / \tau_{\mathrm{H}}\right)\right],
$$

where $H_{\mathrm{PEG}}^{\infty}$ is the brush extension in the limit of infinite $d$ and is set equal to the value of $H_{\mathrm{PEG}}$ obtained for the uncompressed brush. This is justified, because the grafting density of the proximal PEG brush is highly reproducible and because the brush extension exhibits only weak dependence on the grafting density. ${ }^{40}$ The adjustable parameter $\tau_{\mathbf{H}}$ denotes the characteristic length scale of the saturation. Similarly, the $d$-dependence of the power law exponent $n$ is modeled such that its value converges to that of the unperturbed brush $(n=2)$ for large separations:

$$
n(d)=n_{0}+\left(2-n_{0}\right) \cdot\left[1-\exp \left(-d / \tau_{n}\right)\right],
$$

where $\tau_{n}$ denotes the saturation length and $n_{0}$ by construction is the extrapolated value of $n$ for $d=0$. The $d$-dependence of the roughness (or fluctuation amplitude) $\zeta_{\mathrm{D}}$ of the distal lipid layer, is modeled in a more generic manner using a second-order polynomial:

$$
\zeta_{\mathrm{D}}(d)=b_{0}+b_{1} d+b_{2} d^{2}
$$

with adjustable parameters $b_{0}, b_{1}$, and $b_{2}$. To simultaneously fit the adjustable parameters of the common model to a set of experimental reflectivity curves (see Fig. 3A and 5A), we followed our previous approach ${ }^{36}$ and utilized a procedure specified in the ESI. $\dagger$ Estimates of the statistical parameter errors, corresponding to the 95\% (two-sigma) confidence interval are presented in Tables 1-3 in square brackets. Note, however, that these estimates are valid only within the framework of a "perfect model", characterized by a reduced chi-square close to unity $\left(\chi_{\text {red }}^{2} \approx 1\right)$. In view of significant additional contributions due to systematic errors, alternative error estimates are provided in the tables next to the parameter values. They approximately reflect the variation of the obtained parameters throughout the evolution and refinement of the above-described model description, i.e., they reflect the robustness of the parameters with respect to the model, and we therefore consider them more meaningful.

\section{Results and discussion}

\subsection{Structure of a single uncompressed brush}

Fig. 3A shows reflectivity curves (symbols) from a single, uncompressed brush (see Fig. 1B) with $N=114$ and $f=10 \%$, in $\mathrm{D}_{2} \mathrm{O}, 4 \mathrm{MW}, \mathrm{SMW}$, and $\mathrm{H}_{2} \mathrm{O}$. Solid lines represent simulated intensities for the best matching model parameters. The corresponding volume fraction profiles $\Phi(\mathrm{z})$ of $\mathrm{Si}, \mathrm{SiO}_{2}$, hydrocarbon chains $\left(\Phi_{\mathrm{HC}}=\Phi_{\mathrm{OTS}}+\Phi_{\mathrm{CH}}\right)$, headgroups, PEG, and water are shown in Fig. 3B. Apart from the roughness parameters $\zeta$, which are no larger than $12 \AA$ and given in the ESI, $\dagger$ all important model parameters are summarized in Table 1 . The thickness of the oxide layer $\left(d_{\mathrm{SiO}_{2}} \approx 18 \AA\right)$, the significant fraction of hydration species in the oxide $\left(\Phi_{\mathrm{w}}^{\mathrm{SiO}_{2}} \approx 0.16\right)$, and the thickness of the combined hydrocarbon layer $\left(d_{\mathrm{HC}}=d_{\mathrm{OTS}}+d_{\mathrm{CH}} \approx 35 \AA\right)$ are consistent with earlier studies using similar preparation

\begin{tabular}{|c|c|c|c|c|c|c|c|c|}
\hline Parameter & $d_{\mathrm{SiO}_{2}}(\AA)$ & $\Phi_{\mathrm{w}}^{\mathrm{SiO}_{2}}$ & $d_{\mathrm{OTS}}(\AA)$ & $D_{\mathrm{CH}}=d_{\mathrm{CH}}(\AA)$ & $d_{\mathrm{HG}}(\AA)$ & $D_{\mathrm{HG}}(\AA)$ & $D_{\text {PEG }}(\AA)$ & $H_{\mathrm{PEG}}^{\infty}(\AA)$ \\
\hline Value & $\begin{array}{l}18 \pm 2 \\
{[0.2]}\end{array}$ & $\begin{array}{l}0.16 \pm 0.05 \\
{[0.01]}\end{array}$ & $\begin{array}{l}22 \pm 2 \\
{[0.3]}\end{array}$ & $\begin{array}{l}13 \pm 1 \\
{[0.1]}\end{array}$ & $\begin{array}{l}10 \pm 5 \\
{[1]}\end{array}$ & $\begin{array}{l}5 \pm 1 \\
{[0.1]}\end{array}$ & $\begin{array}{l}11 \pm 1 \\
{[0.1]}\end{array}$ & $\begin{array}{l}110 \pm 5 \\
{[1]}\end{array}$ \\
\hline
\end{tabular}
protocols. $^{35,36}$ The thickness of the hydrated headgroup layer, which was neglected in our previous studies, is $d_{\mathrm{HG}} \approx 10 \AA$.

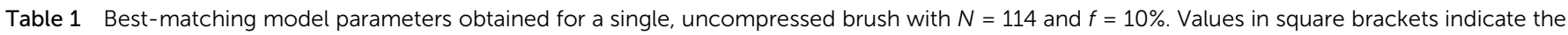
purely statistical parameter errors corresponding to a two-sigma confidence interval 
Table 2 Best-matching separation-independent model parameters obtained for two interacting brushes with $N=114$ and $f=10 \%$. Values in square brackets indicate the purely statistical parameter errors corresponding to a two-sigma confidence interval

\begin{tabular}{lllllll}
\hline Parameter & $d_{\mathrm{SiO}_{2}}(\AA)$ & $\Phi_{\mathrm{w}}^{\mathrm{SiO}_{2}}$ & $d_{\mathrm{OTS}}(\AA)$ & $d_{\mathrm{CH}, \mathrm{P}}=D_{\mathrm{CH}, \mathrm{P}} \approx D_{\mathrm{CH}, \mathrm{D}}=d_{\mathrm{CH}, \mathrm{D}}(\AA)$ & $D_{\mathrm{HG}, \mathrm{P}} \approx D_{\mathrm{HG}, \mathrm{D}}(\AA)$ & $5 \pm 1$ \\
\hline Value & $8 \pm 4$ & $<0.10$ & $14 \pm 1$ & $13 \pm 1$ & {$[0.1]$} & $11 \pm 1$
\end{tabular}

The extension of the uncompressed brush $\left(H_{\mathrm{PEG}}^{\infty}=110 \AA\right)$, is similar to the $103 \AA$ reported previously for the same sample formulation. ${ }^{36}$ Altogether, the agreement between obtained results and earlier reports demonstrates the reproducibility of the sample preparation procedure described in the methods section. The grafting density $\sigma$ follows from $D_{\mathrm{PEG}}$ as $\sigma=D_{\mathrm{PEG}} /$ $\left(N v_{\mathrm{EG}}\right) \approx 13 \times 10^{-4} \AA^{-2}$, where $D_{\mathrm{PEG}}$ is the PEG amount per area obtained using eqn (5).

\subsection{Pressure-distance curves of interacting brushes}

The interaction between extended surfaces across water is typically described in terms of pressure-distance curves, which relate the interaction pressure $\Pi$ to the water layer thickness $D_{\mathrm{w}}$. For fixed temperature $T$ and ambient pressure $p, \Pi$ represents the derivative of the Gibbs free energy $G$ per unit area $A$ with respect to $D_{\mathrm{w}}$,

$$
\Pi\left(D_{\mathrm{w}}\right)=-\frac{1}{A}\left(\frac{\mathrm{d} G}{\mathrm{~d} D_{\mathrm{w}}}\right)_{T, p}
$$

Pressure-distance relations can be determined by subjecting interacting surfaces to dehydrating pressures of known magnitude. ${ }^{41,42}$ So-called equivalent pressures can be exerted for instance by controlling the ambient relative humidity $h_{\text {rel }}$, in which case

$$
\Pi\left(h_{\mathrm{rel}}\right)=-\frac{k_{\mathrm{B}} T}{v_{\mathrm{w}}} \ln \left(h_{\mathrm{rel}}\right),
$$

where $v_{\mathrm{w}}$ denotes the volume of a water molecule. Fig. 4 shows pressure-distance curves of various interacting PEG brushes in this double-monolayer architecture obtained by ellipsometry, where the interaction pressure $\Pi$ was calculated from the measured relative humidity according to eqn (12). Results are compared for various polymer lengths $(22 \leq N \leq 114)$ and PEG-lipid mole fraction $(0 \leq f \leq 10 \%)$. The grafting densities can be estimated as $\sigma=f / A_{\text {lip }}$, where $A_{\text {lip }}=47 \AA^{2}$ is the area per DSPC molecule at $35 \mathrm{mN} \mathrm{m}^{-1}$ (ref. 43). As shown previously, this approximation is relatively good for $f \lesssim 10 \%$ (ref. 36). However, for $N=114$ and $f=10 \%$, the densest brush system studied here, the grafting density measured by NR $\left(\sigma=13 \times 10^{-4} \AA^{-2}\right.$, see previous subsection) is somewhat lower than $f / A_{\text {lip }}=21 \times 10^{-4} \AA^{-2}$, consistent with ref. 36 and presumably due to the significant lateral repulsion of the overlapping polymer chains. Having this in mind, the relative overlaps for the studied brushes approximately span the range $0 \leq \sigma / \sigma_{\mathrm{OT}} \leq 6$, where $\sigma_{\mathrm{OT}}=$ $R_{\mathrm{F}}{ }^{-2}$ is the overlap threshold and $R_{\mathrm{F}}=a N^{3 / 5}$ the Flory radius. With that, both mushroom $\left(\sigma / \sigma_{\mathrm{OT}} \ll 1\right)$ and dilute brush $\left(\sigma / \sigma_{\mathrm{OT}} \gtrsim 1\right)$ regimes are covered. As pointed out by Szleifer, ${ }^{44}$ the AdG model ${ }^{8,9}$ describes brush interactions only for dense enough cases $\left(\sigma / \sigma_{\mathrm{OT}} \gg 1\right)$.

Fig. 4A shows pressure-distance curves for various $N$ and a constant PEG-lipid mole fraction of $f=10 \%$. In panel B, $f$ is varied for a constant polymer length $N=114$. For all brush parameters $\Pi$ is positive and increases with decreasing $D_{\mathrm{w}}$, meaning that the interaction is repulsive and work must be performed in order to bring the surfaces closer. Pure DSPC surfaces exhibit the characteristic exponential decay of the PC lipid hydration repulsion with a decay length of about $\lambda_{\text {hyd }}=$ $0.3 \mathrm{~nm}$ (ref. 45 and 46). The dashed straight lines in Fig. 4A and B indicate an exponential fit with $\lambda_{\text {hyd }}=0.28 \mathrm{~nm}$. In the presence of PEG brushes a systematic increase of the repulsion with increasing $N$ and $f$ is observed. For dense-enough brushes $\left(\sigma / \sigma_{\mathrm{OT}} \gtrsim 5\right)$, for which the repulsion is dominated by the polymers and the hydration repulsion between the grafting surfaces becomes negligible, all pressure distance curves nearly overlap when they are plotted versus a reduced water layer thickness, $D_{\mathrm{w}} /(2 N \sigma)$, which is normalized by the overall grafted PEG amount (Fig. 4C). This result indicates that in the pressure range covered by the ellipsometry measurements the PEG layer jointly formed by the two interacting brushes approximately behaves like an ideal osmotic medium in which only the monomer density matters irrespective of the polymer connectivity. Deviations from this behavior become significant at larger hydration levels $\left(D_{\mathrm{w}} /(2 N \sigma) \gtrsim 100 \AA^{3}\right.$ per monomer). It should be noted that $h_{\text {rel }}$ can only be measured and controlled reliably with a certain precision (see Methods section), which in the present study poses a lower detection limit for interaction pressures of $\Pi \gtrsim 50$ bar.

Table 3 Best-matching separation-dependent model parameters obtained for two interacting brushes with $N=114$ and $f=10 \%$. Values in square brackets indicate the purely statistical parameter errors corresponding to a two-sigma confidence interval. They are not available for the secondary

\begin{tabular}{|c|c|c|c|c|c|c|c|}
\hline$d(\AA ̊)$ & $D_{\mathrm{w}}(\AA)$ & $x$ & $\zeta_{\mathrm{D}}(\AA)$ & $H_{\mathrm{PEG}}(\AA)$ & $n$ & $\xi$ & $I$ \\
\hline $\begin{array}{l}99 \pm 2 \\
{[0.4]}\end{array}$ & $\begin{array}{l}80 \pm 2 \\
{[0.3]}\end{array}$ & $0.45 \pm 0.05$ & $\begin{array}{l}10 \pm 1 \\
{[0.1]}\end{array}$ & $\begin{array}{l}102 \pm 10 \\
{[2]}\end{array}$ & $\begin{array}{l}1.1 \pm 0.2 \\
{[0.1]}\end{array}$ & $0.85 \pm 0.5$ & $0.27 \pm 0.05$ \\
\hline $\begin{array}{l}135 \pm 2 \\
{[0.4]}\end{array}$ & $\begin{array}{l}116 \pm 2 \\
{[0.3]}\end{array}$ & $0.61 \pm 0.05$ & $\begin{array}{l}10 \pm 1 \\
{[0.1]}\end{array}$ & $\begin{array}{l}107 \pm 5 \\
{[1]}\end{array}$ & $\begin{array}{l}1.3 \pm 0.2 \\
{[0.1]}\end{array}$ & $0.91 \pm 0.5$ & $0.15 \pm 0.02$ \\
\hline $\begin{array}{l}170 \pm 2 \\
{[0.2]}\end{array}$ & $\begin{array}{l}150 \pm 2 \\
{[0.1]}\end{array}$ & $0.77 \pm 0.05$ & $\begin{array}{l}10 \pm 1 \\
{[0.1]}\end{array}$ & $\begin{array}{l}109 \pm 5 \\
{[1]}\end{array}$ & $\begin{array}{l}1.4 \pm 0.2 \\
{[0.1]}\end{array}$ & $0.95 \pm 0.5$ & $0.06 \pm 0.02$ \\
\hline
\end{tabular}
quantities $x, \xi$, and $I$ 

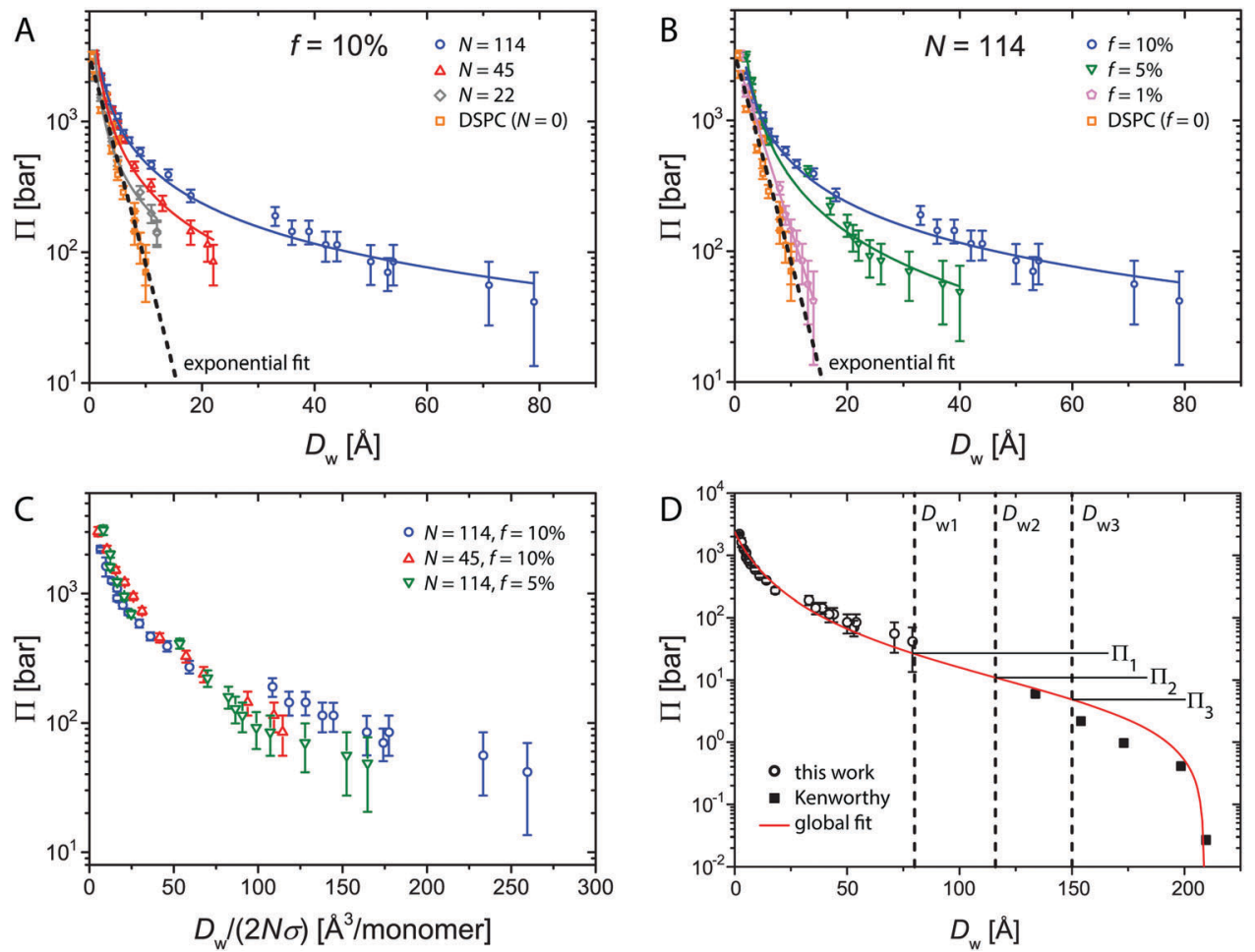

Fig. 4 (A and B) Pressure-distance curves obtained by ellipsometry for interacting polymer brushes (A) with constant PEG-lipid mole fraction $f=10 \%$ and various polymerization degrees $N$ and (B) with constant $N=114$ and various values of $f$. Solid lines are empirical power-law fits that serve to guide the eye. Dashed lines indicate an exponential fit to the data points for pure DSPC, see main text. (C) Interaction pressure for brushes with $\sigma / \sigma_{\mathrm{O}} \gtrsim 5$ plotted versus the reduced water layer thickness $D_{w} /(2 N \sigma)$. (D) Pressure-distance curve for brushes with $f=10 \%$ and $N=114$ plotted together with the one obtained previously by Kenworthy et $a . .^{15}$ for the same formulation in the limit of low interaction pressures. The solid line is a simultaneous fit to both data sets based on the AdG model. Vertical dashed lines indicate the water layer thicknesses $D_{\mathrm{w} 1}, D_{\mathrm{w} 2}$, and $D_{\mathrm{w} 3}$ for which NR was measured. According to the fit they correspond to interaction pressures $\Pi_{1} \approx 25$ bar, $\Pi_{2} \approx 10$ bar, $\Pi_{3} \approx 5$ bar, respectively, indicated with horizontal lines.

A very similar system, composed of self-assembled DSPC/PEGlipid mixed multilayers in water, has been previously investigated by Kenworthy et al. ${ }^{15}$ They obtained pressure distance curves in the limit of low interaction pressures by the addition of hydrophilic polymers that compete for the hydration water. In Fig. $4 \mathrm{D}$ we plot our data points for $f=10 \%$ and $N=114$ together with the data points of Kenworthy for the same formulation as a function of $D_{\mathrm{w}}$. For this purpose, the equivalent thickness of the dry double monolayers, which was measured in the present work by NR as $D_{2 \mathrm{ML}} \approx 58 \AA$ (see further below), was subtracted from the lamellar periodicities reported in their work. It is seen that the data points of Kenworthy et al. virtually constitute a continuous extension of our data points to larger separations, i.e., lower pressures. This indicates that the self-assembled multilayers studied by Kenworthy et al. locally exhibit architectures similar to the well-defined monolayer structures characterized here. Lyngs Hansen et al. ${ }^{47}$ have scrutinized the applicability of the AdG model to PEG brushes of various parameters and concluded that the brush densities corresponding to the data by Kenworthy et al. in Fig. 4D are marginally high enough for the AdG model:

$$
\Pi(D)=\alpha\left[\left(\frac{L_{0}}{D / 2}\right)^{9 / 4}-\left(\frac{D / 2}{L_{0}}\right)^{3 / 4}\right], \quad D<2 L_{0}
$$

where $\alpha$ is a temperature-dependent pre-factor, $L_{0}$ is the uncompressed brush thickness in the AdG approximation, and $D$ is the interaction distance. The solid curve in Fig. 4D represents a simultaneous fit of eqn (13) to our data points and to those by Kenworthy et al. To empirically account for the dry volume of PEG, which becomes important at low hydration, the interaction distance is not set equal to the surface separation, but as $D=D_{\mathrm{w}}+\Delta d$, with an adjustable parameter $\Delta d$. The fit yields $\Delta d=13 \AA$ and $L_{0}=111 \AA$, where the latter is remarkably close to the unperturbed brush extension determined above, $H_{\mathrm{PEG}}^{\infty}=110 \AA$ A. While this result is reassuring, the obtained simultaneous fit to all data points also yields an empirical description of the interaction pressure in the entire hydration range. This is useful especially because our NR results include very highly hydrated states (indicated in Fig. 4D with dashed vertical lines, see next section), which are not covered by the present ellipsometry measurements.

\subsection{Distance-dependent structures of interacting brushes}

Fig. 5A shows neutron reflectivity curves of interacting lipidanchored PEG brushes (see Fig. 1C) with $f=10 \%$ and $N=114$ for various humidity levels. The dehydrating pressure $\Pi$ depends on $h_{\text {rel }}$ according to eqn (12). As pointed out above, $h_{\text {rel }}$ can be measured only to certain precision, so that there is a lower detection limit for $\Pi$. Nonetheless, much lower 

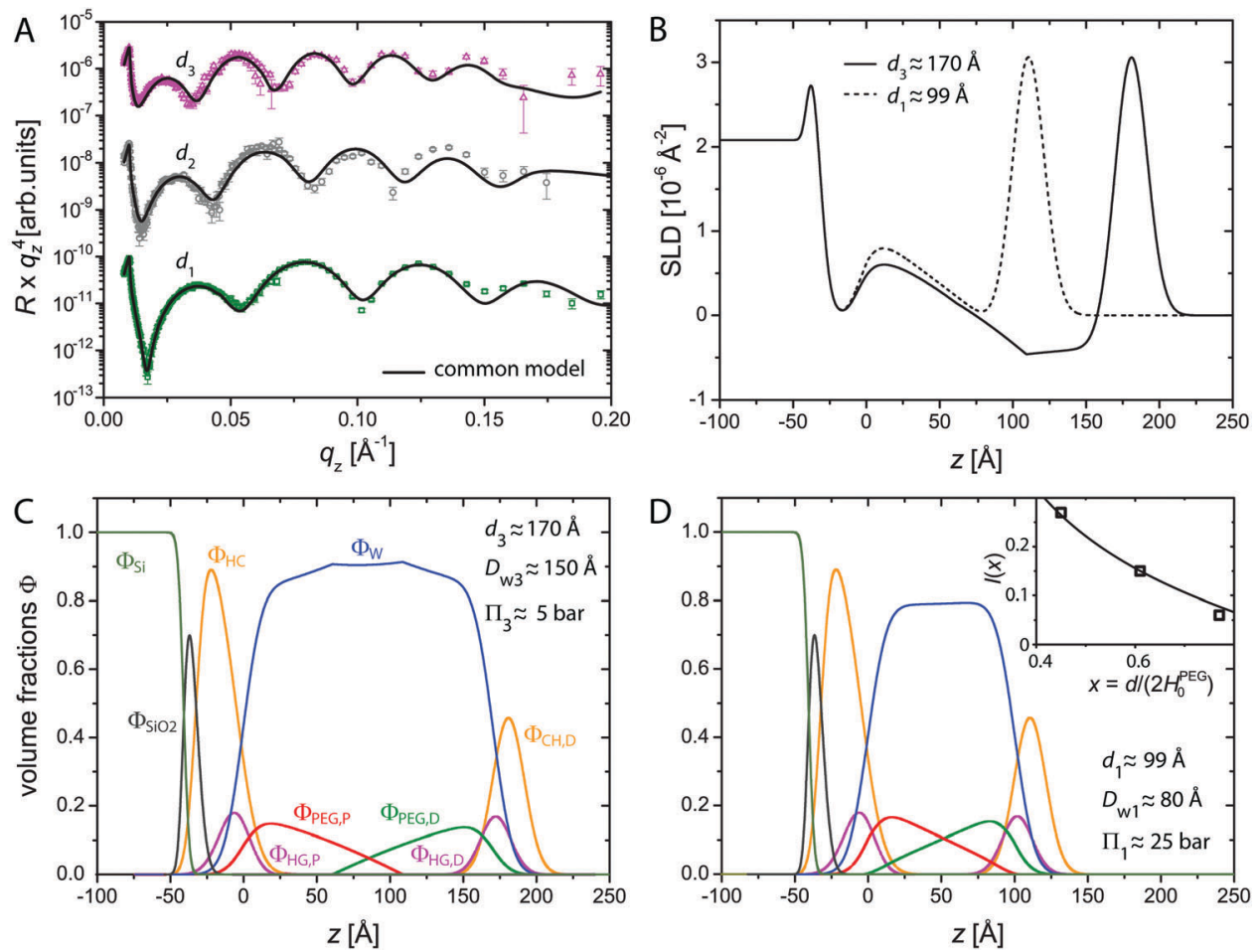

Fig. 5 (A) Neutron reflectivity curves (symbols) of interacting two brushes with $f=10 \%$ and $N=114$ obtained for various relative humidities corresponding to grafting surface separations of $d_{1}=101 \AA, d_{2}=136 \AA$, and $d_{3}=171 \AA$. Solid lines indicate the theoretical reflectivity curves according to the best-matching parameters in the common model. The reduced chi-square deviation is $\chi_{\text {red }}{ }^{2}=4.9$. (B) Corresponding scattering length density (SLD) profiles for $d_{1}$ and $d_{3}$, respectively. (C and D) Corresponding volume fraction profiles for $d_{3}$ and $d_{1}$, respectively. Inset in panel D: Brush interpenetration as a function of the reduced surface separation $x$. The solid line represents a scaling law proposed by Murat and Grest. ${ }^{12}$

(albeit immeasurable) dehydration pressures can be realized in a humidity chamber using suitable bath temperatures, at least transiently. The corresponding extremely high values of $h_{\text {rel }}$, which come close to the condensation limit, lead to very strong water uptake of the interacting brushes, i.e. to large grafting surface separations $d$. The latter, in turn, are precisely encoded in the $q_{z}$-positions of the minima in the reflectivity curves (see Fig. 5A). With that, NR puts us in the position to investigate the structure of the interacting brushes in a wide separation range. The sold lines in Fig. 5A indicate the simulated reflectivity curves corresponding to the best-matching simultaneous model in terms of the $d$-independent and $d$-dependent parameters specified in the methods section. Fig. 5C and D show the corresponding sample structures at humidity 3 (the highest) and 1 (the lowest), respectively, in terms of the volume fraction profiles $\Phi_{i}(z)$. It is seen immediately that the sample exhibits a highly ordered, layered, and symmetrical structure with the double-monolayer architecture "as intended". Fig. 5B shows the obtained SLD profiles at humidities 3 and 1 . We recall that the proximal brush is deuterated, so that NR can distinguish between the PEG distributions belonging proximal and distal brushes. In particular, the measurements are very sensitive to the brush interpenetration. Namely, the SLD gradient around the midplane is much sharper for weakly interpenetrating brushes than for strongly penetrating ones. In the following, the best-matching model parameters will be discussed.
The grafting surface separation $d$ plays a distinct role as it acts as "reaction coordinate" of the interaction. For humidities 1,2 , and 3, respectively, $d_{1}=99 \AA, d_{2}=135 \AA$, and $d_{3}=170 \AA$ are obtained. According to eqn (5), the corresponding water layer thicknesses via the respective water distribution profiles follow as $D_{\mathrm{w} 1}=80 \AA, D_{\mathrm{w} 2}=116 \AA$ and $D_{\mathrm{w} 3}=150 \AA$, indicated in Fig. $4 \mathrm{D}$ as dashed vertical lines. The corresponding interaction pressures according to eqn (13) with the best-matching parameters of $\alpha$, $L_{0}$ and $\Delta d$ (solid line in Fig. $4 \mathrm{D}$ ) are $\Pi_{1} \approx 25$ bar, $\Pi_{2} \approx 10$ bar, $\Pi_{3} \approx 5$ bar, respectively.

The $d$-independent parameters characterizing $\mathrm{SiO}_{2}$, OTS, as well as proximal and distal monolayers (Table 2) are in satisfactory agreement with those obtained for the single, uncompressed brush (Table 1) and those reported previously for the same preparation protocol. ${ }^{36}$ Differences can be attributed to the history of the silicon blocks and to ensuing differences in the efficacy of the OTS deposition. As perceptible already from the overall symmetrical shape of the profiles shown in Fig. 5C and $\mathrm{D}$ and as encoded in $D_{\mathrm{CH}, \mathrm{P}} \approx D_{\mathrm{CH}, \mathrm{P}} \approx 13 \AA$, the obtained lipid and brush amounts in the proximal and distal monolayers are almost identical. This implies that the transfer ratio for the distal monolayer during preparation was similar to the one for the proximal monolayer, which is known to be close to $100 \%$ (see Methods section). The same by construction also holds for $D_{\mathrm{HG}, \mathrm{P}}$ and $D_{\mathrm{HG}, \mathrm{D}}$, as well as for $D_{\mathrm{PEG}, \mathrm{P}}$, and $D_{\mathrm{PEG}, \mathrm{D}}$. This result confirms the quality of the employed LS/LB transfer involving a $90^{\circ}$ rotation (see Methods section) and justifies the application 
of theoretical models assuming symmetrical interaction scenarios. The overall equivalent thickness of the two brushdecorated monolayers is $D_{2 \mathrm{ML}}=D_{\mathrm{HC}, \mathrm{P}}+D_{\mathrm{HC}, \mathrm{D}}+D_{\mathrm{HG}, \mathrm{P}}+D_{\mathrm{HG}, \mathrm{D}}+$ $D_{\mathrm{PEG}, \mathrm{P}}+D_{\mathrm{PEG}, \mathrm{P}} \approx 2\left(D_{\mathrm{HC}, \mathrm{P}}+D_{\mathrm{HG}, \mathrm{P}}+D_{\mathrm{PEG}, \mathrm{P}}\right) \approx 58 \AA$.

The best-matching $d$-dependent model parameters are summarized in Table 3. We first have a look at the roughness of the distal lipid surface, $\zeta_{\mathrm{D}}(d)$. The obtained parameters $b_{0}, b_{1}$, and $b_{2}$ (eqn (10)) correspond to a virtually $d$-independent roughness of $\zeta_{\mathrm{D}} \approx 10 \AA$ throughout the entire separation range $d_{1}<d<d_{3}$. The obtained roughness is somewhat larger than that of a typical lipid monolayer at an air/water interface; however this result is in line with the observation that lipopolymers systematically increase the roughness of lipid monolayers. ${ }^{48}$ As seen in Fig. 5B, the roughness has its manifestation in a significant broadening of the of the lipid headgroup and chain volume fraction profiles $\Phi_{\mathrm{HG}, \mathrm{D}}(z)$ and $\Phi_{\mathrm{CH}, \mathrm{D}}(z)$, whose full-widths at half maximum (FWHM) would be as low as $d_{\mathrm{HG}} \approx 10 \AA$ and $d_{\mathrm{CH}} \approx 13 \AA$, respectively, without roughness. The power law exponent of the PEG brushes, $n(d)$, according to the obtained parameters $\tau_{n}$ and $n_{0}$ (eqn (9)) exhibits a weak separation-dependence resulting in $n\left(d_{1}\right) \approx 1.1$, $n\left(d_{2}\right) \approx 1.3$, and $n\left(d_{3}\right) \approx 1.4$. Such a change from a parabolic shape $(n=2)$ to a more linear shape $(n=1)$ upon compression is in qualitative agreement with simulations results by Grest $^{13}$ and the experiments by Mulder and Kuhl. ${ }^{19}$ The interpenetration of the opposing PEG brushes strongly increases with decreasing surface separation (see Fig. 5D). The amount of interpenetration, $I(d)$, is determined as: ${ }^{12}$

$$
I(d)=\frac{1}{D_{\mathrm{PEG}, \mathrm{P}}} \int_{d / 2}^{\infty} \Phi_{\mathrm{PEG}, \mathrm{P}}(z, d) \mathrm{d} z=\frac{1}{D_{\mathrm{PEG}, \mathrm{D}}} \int_{-\infty}^{d / 2} \Phi_{\mathrm{PEG}, \mathrm{D}}(z, d) \mathrm{d} z .
$$

The resulting values, $I\left(d_{1}\right) \approx 0.27, I\left(d_{2}\right) \approx 0.15$, and $I\left(d_{3}\right) \approx 0.06$ are plotted in the inset of Fig. 5D as a function of the reduced surface separation $x=d /\left(2 H_{\mathrm{PEG}}^{\infty}\right)$. Based on an estimation for the brush overlap range by Witten et al., ${ }^{49}$ Murat and Grest $^{12}$ proposed a scaling law for $I(x)$,

$$
I(x) \propto x^{-4 / 3}\left(1-x^{3}\right) .
$$

The solid line in the inset of Fig. 5D indicates this scaling law for a suitable pre-factor, and it is seen that it roughly describes our experimental results. Because of the pronounced interpenetration, the compression of the brushes with decreasing separation is weak. This behavior is encoded in the weak $d$-dependence of the brush extension $H_{\mathrm{PEG}}(d)$. The best-matching saturation length is as low as $\tau_{\mathrm{H}} \approx 60 \AA$ and according to eqn (8) coincides with $H_{\mathrm{PEG}}\left(d_{1}\right) \approx 102 \AA, H_{\mathrm{PEG}}\left(d_{2}\right) \approx 107 \AA$, and $H_{\mathrm{PEG}}\left(d_{3}\right) \approx$ $109 \AA$. Brush compression is commonly quantified in terms of the compression parameter, ${ }^{10} \xi(d)=h_{\mathrm{rms}}(d) / h_{\mathrm{rms}}(\infty)$, where

$$
\begin{aligned}
h_{\mathrm{rms}}^{2}(d) & =\frac{1}{D_{\mathrm{PEG}, \mathrm{P}}} \int_{-\infty}^{\infty} z^{2} \Phi_{\mathrm{PEG}, \mathrm{P}}(z, d) \mathrm{d} z \\
& =\frac{1}{D_{\mathrm{PEG}, \mathrm{D}}} \int_{-\infty}^{\infty}(d-z)^{2} \Phi_{\mathrm{PEG}, \mathrm{D}}(z, d) \mathrm{d} z
\end{aligned}
$$

is the separation-dependent second moment of the single-sided PEG distributions. For $d_{1}, d_{2}$, and $d_{3}$ we obtain $\xi\left(d_{1}\right) \approx 0.85$, $\xi\left(d_{2}\right) \approx 0.91$, and $\xi\left(d_{3}\right) \approx 0.95$, respectively. This level of compression is much weaker than that predicted by analytical SCF theory, which does not account for interpenetration $\left(\xi_{\mathrm{SCF}}\left(d_{1}\right)=0.57, \xi_{\mathrm{SCF}}\left(d_{2}\right)=0.74\right.$, and $\left.\xi_{\mathrm{SCF}}\left(d_{3}\right)=0.89\right)$. While numerical SCF calculations predict significant interpenetration close to the overlap threshold $\left(\sigma / \sigma_{\mathrm{OT}} \gtrsim 1\right)$ (ref. 10), strong interpenetration comparable to our experimental results as well as the corresponding weak compression are reported only in simulation-based theoretical studies. ${ }^{12-14}$

\subsection{Discussion}

The AdG model, despite making strong approximations describes the experimentally determined pressure-distance curve in Fig. 4D surprisingly well over several orders of magnitude both in pressure and distance, when introducing an empirical, effective interaction distance. In contrast, analytical descriptions like AdG and SCF are less powerful in predicting conformations of interacting brushes. Interpenetration comparable to our experimental results is only observed with rigorous, simulation-based theoretical approaches. Accurate theoretical prediction of brush interpenetration is important because it has strong impact on adhesion forces in chemically dissimilar brushes and on shear friction. ${ }^{7}$ Our work therefore motivates further simulation work, especially on dilute brushes and on strongly dehydrated brushes where conformations are no longer governed by a maximization of configurational entropy alone, but also by their molecular-level interaction with water. The latter will require refined treatments of monomer-monomer interactions but may more accurately reproduce pressure-distance curves in the limit of low hydration. In the future, valuable experimental data on brush conformations can be expected from element-specific structural investigations, ${ }^{50}$ which are sensitive, for instance, to the endpoint-distributions in interacting brushes. ${ }^{24}$

\section{Conclusions}

We have prepared pairs of planar hydrophilic polymer brushes with well-defined parameters in terms of polymerization degree and grafting density. They interact across thin water layers while the grafting surface separation can be adjusted via the application of defined dehydrating pressures. We have demonstrated that this architecture enables the determination of interaction forces and of the separation-dependent internal structures of the interacting brushes at high resolution by neutron reflectometry. The combination of pressure-distance curves and brush conformations in terms of interpenetration and compression yields the most comprehensive experimental description of interacting polymer brushes so far. The results may therefore serve as comparison for refined theoretical models and computer simulations. The presented approach is not limited to polymer brushes but generally applicable to interacting soft interfaces. 


\section{Conflicts of interest}

There are no conflicts of interest to declare.

\section{Acknowledgements}

The authors thank Institut Laue-Langevin (ILL) for beam time allocation and the ILL/PSCM laboratories for support during sample preparation and pre-characterization. We thank Philipp Gutfreund for support during beamtimes and Peter Fratzl and Georgi Gochev for insightful comments. Financial support by the Max Planck Society and by Deutsche Forschungsgemeinschaft (DFG) via Emmy-Noether grant (SCHN 1396/1) is gratefully acknowledged. Open Access funding provided by the Max Planck Society.

\section{References}

1 H. Chen, L. Yuan, W. Song, Z. Wu and D. Li, Prog. Polym. Sci., 2008, 33, 1059-1087.

2 E. P. K. Currie, W. Norde and M. A. C. Stuart, Adv. Colloid Interface Sci., 2003, 100, 205-265.

3 B. Kolaric, S. Förster and R. v. Klitzing, Adsorption and Nanostructure, Springer, 2001, pp. 195-199.

4 U. Raviv, S. Giasson, N. Kampf, J.-F. Gohy, R. Jérôme and J. Klein, Nature, 2003, 425, 163-165.

5 S. Weinbaum, J. M. Tarbell and E. R. Damiano, Annu. Rev. Biomed. Eng., 2007, 9, 121-167.

6 P. C. Y. Lau, T. Lindhout, T. J. Beveridge, J. R. Dutcher and J. S. Lam, J. Bacteriol., 2009, 191, 6618-6631.

7 T. Kreer, M. H. Müser, K. Binder and J. Klein, Langmuir, 2001, 17, 7804-7813.

8 S. Alexander, J. Phys. France, 1977, 38, 983-987.

9 P. G. de Gennes, Adv. Colloid Interface Sci., 1987, 27, 189-209.

10 M. D. Whitmore and R. Baranowski, Macromol. Theory Simul., 2005, 14, 75-95.

11 C. M. Wijmans, E. B. Zhulina and G. J. Fleer, Macromolecules, 1994, 27, 3238-3248.

12 M. Murat and G. S. Grest, Phys. Rev. Lett., 1989, 63, 1074-1077.

13 G. S. Grest, Phys. Rev. Lett., 1996, 76, 4979-4982.

14 R. 1. Toral, A. Chakrabarti and R. Dickman, Phys. Rev. E: Stat. Phys., Plasmas, Fluids, Relat. Interdiscip. Top., 1994, 50, 343-348.

15 A. K. Kenworthy, K. Hristova, D. Needham and T. J. McIntosh, Biophys. J., 1995, 68, 1921-1936.

16 T. L. Kuhl, D. E. Leckband, D. D. Lasic and J. N. Israelachvili, Biophys. J., 1994, 66, 1479-1488.

17 T. W. Kelley, P. A. Schorr, K. D. Johnson, M. Tirrell and D. Frisbie, Macromolecules, 1998, 31, 4297-4300.

18 W. A. Hamilton, G. S. Smith, N. A. Alcantar, J. Majewski, R. G. Toomey and T. L. Kuhl, J. Polym. Sci., Part B: Polym. Phys., 2004, 42, 3290-3301.

19 D. J. Mulder and T. L. Kuhl, Soft Matter, 2010, 6, 5401-5407.

20 T. P. Russell, Chimia, 1990, 44, 312-315.

21 J. Als-Nielsen and D. McMorrow, Elements of Modern X-Ray Physics, Wiley, Chichester, 2001.
22 J. Daillant and A. Gibaud, X-ray and Neutron Reflectivity: Principles and Applications, Springer, Berlin, 2009.

23 D. D. Lasic and F. J. Martin, Stealth liposomes, CRC press, 1995.

24 E. Schneck, I. Rodriguez-Loureiro, L. Bertinetti, E. Marin, D. Novikov, O. V. Konovalov and G. Gochev, J. Phys. D, 2017, 50, 104001.

25 Z. Wei and S. W. Prescott, Curr. Opin. Colloid Interface Sci., 2015, 20, 253-260.

26 L. Tamm and H. M. McConnell, Biophys. J., 1984, 47, 105-113.

27 R. M. A. Azzam and N. M. Bashara, Ellipsometry and polarized light, Elsevier Science Publishing Co., Inc., NorthHolland, sole distributors for the USA and Canada, 1987.

28 S. Adachi, Phys. Rev. B: Condens. Matter Mater. Phys., 1988, 38, 12966-12976.

29 I. H. Malitson, J. Opt. Soc. Am., 1965, 55, 1205-1209.

30 J. E. Wong, F. Rehfeldt, P. Hänni, M. Tanaka and R. v. Klitzing, Macromolecules, 2004, 37, 7285-7289.

31 J. Ruths, F. Essler, G. Decher and H. Riegler, Langmuir, 2000, 16, 8871-8878.

32 R. Reiter, H. Motschmann and W. Knoll, Langmuir, 1993, 9, 2430-2435.

33 J. Katsaras and M. J. Watson, Rev. Sci. Instrum., 2000, 71, 1737-1739.

34 S. A. Tristram-Nagle, Methods Membr. Lipids, 2007, 400, 63-75. 35 E. Schneck, A. Schollier, A. Halperin, M. Moulin, M. Haertlein, M. Sferrazza and G. Fragneto, Langmuir, 2013, 29, 14178-14187. 36 E. Schneck, I. Berts, A. Halperin, J. Daillant and G. Fragneto, Biomaterials, 2015, 46, 95-104.

37 A. P. Dabkowska, J. P. Talbot, L. Cavalcanti, J. R. P. Webster, A. Nelson, D. J. Barlow, G. Fragneto and M. J. Lawrence, Soft Matter, 2013, 9, 7095-7105.

38 E. Loizou, L. Porcar, P. Schexnailder, G. Schmidt and P. Butler, Macromolecules, 2010, 43, 1041-1049.

39 S. T. M. a. T. A. Witten, Macromolecules, 1988, 21, 2610-2619. 40 S. T. Milner, Science, 1991, 251, 905-914.

41 V. A. Parsegian, N. Fuller and R. P. Rand, Proc. Natl. Acad. Sci. U. S. A., 1979, 76, 2750-2754.

42 E. Schneck, Adv. Mater. Interfaces, 2017, 4, 1600349.

43 A. Hermelink and G. Brezesinski, J. Lipid Res., 2008, 49, 1918-1925.

44 I. Szleifer, Curr. Opin. Colloid Interface Sci., 1996, 1, 416-423. 45 L. J. Lis, M. McAlister, N. Fuller, R. P. Rand and V. A. Parsegian, Biophys. J., 1982, 37, 657.

46 E. Schneck, F. Sedlmeier and R. R. Netz, Proc. Natl. Acad. Sci. U. S. A., 2012, 109, 14405-14409.

47 P. Lyngs Hansen, J. A. Cohen, R. Podgornik and V. A. Parsegian, Biophys. J., 2003, 84, 350-355.

48 J. Majewski, T. L. Kuhl, M. C. Gerstenberg, J. N. Israelachvili and G. S. Smith, J. Phys. Chem. B, 1997, 101, 3122-3129.

49 T. A. Witten, L. Leibler and P. A. Pincus, Macromolecules, 1990, 23, 824-829.

50 E. Schneck, E. Scoppola, J. Drnec, C. Mocuta, R. Felici, D. Novikov, G. Fragneto and J. Daillant, Proc. Natl. Acad. Sci. U. S. A., 2016, 113, 9521-9526. 